\section{Defining the path: lung cancer CT screening in Europe}

\author{
Matthew E Callister, ${ }^{1}$ Sam M Janes ${ }^{2}$
}

Since the publication of the National Lung Screening Trial (NLST) in 2011 demonstrating a $20 \%$ reduction in lung cancer-specific mortality by annual low-dose CT (LDCT), ${ }^{1}$ implementation of screening has continued apace in the USA. Other developed nations, perhaps mindful of the potential cost implications of national screening programmes, have been more reticent, with many awaiting the final outcome data from the largest European randomised trial- the Dutch-Belgian Lung Cancer Screening trial (NELSON) study which remains in follow-up. ${ }^{2}$

Despite the convincing mortality reduction in NLST, questions remain about implementation of LDCT screening for lung cancer. Unresolved issues include how to best identify high-risk individuals for screening, what is the most appropriate screening interval to achieve an optimal balance between mortality reduction and cost-effectiveness and how to minimise possible harms associated with screening-particularly overdiagnosis. This issue of Thorax includes two papers which provide important additions to accumulating evidence in this field.

Paci and colleagues ${ }^{3}$ report lung cancer incidence and mortality rates from the Italian Lung Cancer Screening Trial (ITALUNG) randomised lung cancer screening trial conducted in Tuscany, Italy, commencing in 2004. From an invited population of over 71232 subjects aged 55-69 years, 1613 were randomised to four rounds of annual LDCT screening and 1593 to usual care. Like many of the other European screening studies, ITALUNG was not powered to demonstrate mortality reduction in isolation, and pooled analysis with other similarly designed randomised trials is planned.

As expected, lung cancer incidence was higher in the intervention group during the 4-year screening period $(55 \%$ excess diagnoses vs usual care group). However,

Department of Respiratory Medicine, Leeds Teaching Hospitals NHS Trust, Leeds, UK

${ }^{2}$ Lungs for Living Research Centre, UCL Respiratory, University College London, London, UK

Correspondence to Dr Sam M Janes, Lungs for Living Research Centre, UCL Respiratory, University College London, Rayne Building, 5 University Street, London WC1E 6JF, UK; s.janes@ucl.ac.uk this was followed by a compensatory reduction in diagnoses during the 5-year follow-up period, such that there was no significant difference in incidence rates between the groups at the end of the study. After a median follow-up of 9 years, the authors report non-significant reductions of $17 \%$ for overall mortality (rate ratio $(\mathrm{RR})=0.83,95 \% \mathrm{CI} 0.67$ to 1.03$)$, and $30 \%$ for lung cancer-specific mortality $(\mathrm{RR}=0.70,95 \% \mathrm{CI} 0.47$ to 1.03$)$.

The completion of 'catch-up' in lung cancer diagnoses in the non-screened arm is an important finding as it would tend to suggest that there was no significant overdiagnosis from LDCT screening. This is in contrast to the NLST, where there was an $13 \%$ excess in diagnoses in the LDCT arm compared with the chest X-ray (CXR) arm, ${ }^{1}$ with a probability of $18.5 \%$ that any CT screen-detected cancer was overdiagnosed. ${ }^{4}$ This may relate to the shorter follow-up period in NLST (6.5 years compared with 9 years in ITALUNG), and it maybe that with longer follow-up, catch up will be seen in the NLST study also. Alternatively, this may reflect different management strategies for screen-detected nodules-particularly indolent adenocarcinomas manifesting as pure ground glass nodules ( $\mathrm{pGGNs).}$ Analysis of possible overdiagnosis by histological subtype in NLST showed that up to $78.9 \%$ of the (then-termed) bronchioalveolar lung cancers detected by LDCT were overdiagnosed. ${ }^{4}$ The International Early Lung Cancer Action Programme group have demonstrated the safety of continued annual observation for pGGNs in the absence of a solid component. ${ }^{5}$ Neither NLST nor ITALUNG have published nodule management protocols, so it is impossible to assess the extent to which management differed between these studies. Nevertheless, there is increasing evidence suggesting that these indolent lesions may be best observed rather than removed, and future screening studies should consider prospective evaluation of protocols mandating observation only for pGGNs to minimise overdiagnosis.

The larger reductions in lung cancer-specific mortality and all-cause mortality in ITALUNG $(17 \%$ and $30 \%$, respectively) compared with NLST $(6.7 \%$ and $20 \%$, respectively) are discussed by the authors. Possible explanations proposed are the longer follow-up period, and the more advanced stage distribution of lung cancers in the control arm of ITALUNG compared with NLST. Caution must be exercised in assessing non-significant outcomes for which the study was not originally powered. However, the stage distribution in the non-CT screened arms are very different between the two studies. In the usual care arm of ITALUNG (which did not receive CXR screening), only 11\% of patients diagnosed with lung cancer had stage I disease compared with $31 \%$ in the CXR-screened control arm of NLST. The low proportion of usual care patients with stage I disease in Italy reflects data from other European countries published in the International Cancer Benchmarking Partnership (stage I disease $14.0 \%$ in Denmark, $13.5 \%$ in UK). ${ }^{6}$ It may be, therefore, that mortality reduction from LDCT screening maybe augmented in locations with more adverse stage distribution in their background populations, or indeed more simply, in populations not screened with CXRs. The final results of the NELSON trial are therefore keenly awaited in this context. Interestingly, the proportion of patients with stage I disease in the usual care (unscreened) arm of the Prostate Lung Colorectal and Ovarian study of CXR screening for lung cancer was also high at $27 \%{ }^{7}$

The issue of optimal screening interval is addressed by Yousaf-Khan and colleagues from the NELSON group. ${ }^{8}$ Unlike NLST, the NELSON study employed a volumetric-based nodule management protocol, and included increasing screening intervals (of 1 , then 2 then 2.5 years) during the course of the study. The data presented here show the cancer yield of the fourth screening round (performed 2.5 years after the third) according to the results of previous screening rounds and patient characteristics. Outcomes from screening rounds were classed as negative (newly detected nodules $<50 \mathrm{~mm}^{3}$ or previously detected nodules with volume doubling time of $>600$ days), indeterminate (newly detected nodules $50-500 \mathrm{~mm}^{3}$ or volume doubling time (VDT) of 400-600 days) or positive (newly detected nodule $>500 \mathrm{~mm}^{3}$ or VDT $<400$ days).

Of the 5279 participants in the fourth screening round, 3856 had all negative previous rounds, 1342 had had at least one indeterminate previous round (but never a positive) and 81 had a previous positive round. The proportion of these groups with a screen-detected cancer at the fourth round were $0.6 \%$ for those with all previous negatives and $1.6 \%$ for 
those with at least one previous indeterminate result equating to an $\mathrm{OR}$ of 2.7 (95\% CI 1.5 to 5.1 vs negative result). No clinical factors (age, gender, starting age of smoking, smoking status or pack-years smoked) significantly predicted detection of lung cancer in the fourth round.

The low cancer yield following a previous negative round described by the NELSON group matches closely with a similar recent retrospective analysis of NLST. $^{9}$ Of 19066 participants with a negative prevalence scan (first scan), the yield of lung cancer at the first incidence screen (after a 1-year interval) was only $0.34 \%$. Considering the associated harms of overly frequent screening, the authors suggest that increasing the screening interval may be appropriate for those with negative baseline scans.

Extending screening intervals, however, risks lowering the rate of detection of early stage cancers. Importantly, another recent Thorax publication from the NELSON group demonstrated a progressive reduction in the proportion of screen-detected cancers at the earliest stage (stage IATNM seventh edition) with increasing screening interval $(74.1 \%$ following 1 -year interval, $64.9 \%$ following 2-year interval, $47.8 \%$ following 2.5 -year interval). ${ }^{10}$ The survival advantage of diagnosing lung cancer at its earliest (and smallest) stage is highlighted in the eighth edition of TNM classification by the IASLC Lung Cancer Staging Project. Five-year survival rates for stage IA1 (tumour $\leq 1 \mathrm{~cm}$ ), stage IA2 (tumour $1-2 \mathrm{~cm}$ ) and stage IA3 (tumour $2-3 \mathrm{~cm}$ ) were $92 \%, 83 \%$ and $77 \%$, respectively. ${ }^{11}$ The demonstrated reduction in earliest stage disease with increased screening interval may therefore attenuate the benefits demonstrated in NLST.

The combined data from NELSON and NLST therefore suggest that one size may not fit all when selecting a screening interval. A 2.5-year (or even 2-year interval) seems to be associated with a trend to more advanced disease, and therefore may not be appropriate for all participants as it risks losing some of the mortality reduction originally described. However, the very low cancer yield following previous negative rounds may identify a low-risk group where a prolonged interval maybe safe. Those with a previous indeterminate result (even where the nodule in question is subsequently deemed benign) may benefit from ongoing annual screens due to the increased risk that the indeterminate result seems to confer. Tailoring screening interval to lung cancer risk (by virtue of screening history) is therefore one promising option to optimise the benefits of screening while minimising costs and harms. Future studies should therefore look to prospectively evaluate such tailored screening intervals.

A final and intriguing question is why indeterminate nodules, subsequently deemed benign by further evaluation, still seem to confer an increased risk of lung cancer. Some of this may reflect the original NELSON nodule management protocol. In the protocol first described, ${ }^{12}$ all nodules less than $50 \mathrm{~mm}^{3}$ were deemed benign and ignored. However, subsequent analysis from NELSON suggests that this threshold maybe too high when considering newly appearing nodules. Instead, Walter and colleagues propose a lower volume cut-off of $27 \mathrm{~mm}^{3}$ suggesting that nodules ignored according to the protocol at the time may have conferred increased risk. However, the majority of lung cancers diagnosed in round 4 in the current study were detected in a new nodule not previously seen. Why then should benign nodules (many of which subsequently disappear) predict the development of cancer? Do these nodules reflect alveolar inflammation either in direct response to cigarette smoke, or to intercurrent infection commonly seen in patients with chronic obstructive pulmonary disease, and this in turn predisposes to lung cancer? Or could the nodules that appear and disappear in fact be early cancers subsequently cleared by immune-editing mechanisms? In an age when the funders of healthcare are increasingly concerned about how to resource the anticipated explosion in immuno-oncology, are we witnessing this phenomenon for free in serial CT screening scans?

Contributors Both the authors contributed equally to this work.
Competing interests None declared.

Provenance and peer review Commissioned; externally peer reviewed.

(c) Article author(s) (or their employer(s) unless otherwise stated in the text of the article) 2017. All rights reserved. No commercial use is permitted unless otherwise expressly granted.

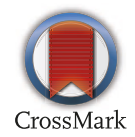

To cite Callister ME, Janes SM. Thorax 2017:72:778-779

Published Online First 19 July 2017

Thorax 2017:72:778-779.

doi:10.1136/thoraxjnl-2017-210268

\section{REFERENCES}

1 Aberle DR, Adams AM, Berg CD, et al. Reduced lungcancer mortality with low-dose computed tomographic screening. N Eng/ J Med 2011;365:1-15.

2 Oudkerk M, Heuvelmans MA. Screening for lung cancer by imaging: the NELSON study. J Belg SoC Radiol 20132014;96:163-6.

3 Paci E, Puliti D, Lopes Pegna A, et al. Mortality, survival and incidence rates in the ITALUNG randomised lung cancer screening trial. Thorax 2017;72:825-31.

4 Patz EF, Pinsky P, Gatsonis C, et al. Overdiagnosis in low-dose computed tomography screening for lung cancer. JAMA Intern Med 2014;174:269-74.

5 Yankelevitz DF, Yip R, Smith JP, et al. CT Screening for Lung Cancer: Nonsolid Nodules in Baseline and Annual Repeat Rounds. Radiology 2015;277:555-64.

6 Walters S, Maringe C, Butler J, et al. Comparability of stage data in cancer registries in six countries: lessons from the International Cancer Benchmarking Partnership. Int J Cancer 2013;132:676-85.

7 Oken MM, Hocking WG, Kvale PA, et al. Screening by chest radiograph and lung cancer mortality: the Prostate, Lung, Colorectal, and Ovarian (PLCO) randomized trial. JAMA 2011;306:1865-73.

8 Yousaf-Khan U, van der Aalst C, de Jong PA, et al. Risk stratification based on screening history: the NELSON lung cancer screening study. Thorax 2017;72:819-24.

9 Patz EF, Greco E, Gatsonis C, et al. Lung cancer incidence and mortality in National Lung Screening Trial participants who underwent low-dose CT prevalence screening: a retrospective cohort analysis of a randomised, multicentre, diagnostic screening trial. Lancet Oncol 2016;17:590-9.

10 Yousaf-Khan U, van der Aalst C, de Jong PA, et al. Final screening round of the NELSON lung cancer screening trial: the effect of a 2.5-year screening interval. Thorax 2017;72:48-56

11 Goldstraw P, Chansky K, Crowley J, et al. The IASLC Lung Cancer Staging Project: Proposals for Revision of the TNM Stage Groupings in the Forthcoming (Eighth) Edition of the TNM Classification for Lung Cancer. J Thorac Oncol 2016;11:39-51.

12 Xu DM, Gietema $\mathrm{H}$, de Koning $\mathrm{H}$, et al. Nodule management protocol of the NELSON randomised lung cancer screening trial. Lung Cancer 2006;54:177-84. 